\title{
Stock price forecasting model analysis based on the wavelet neural network and the data cleaning technology
}

\author{
Xin Sun ${ }^{1}$, Ziqi Tang ${ }^{2}$ and Ziwei Guo ${ }^{3}$ \\ 1Johns Hopkins University, Carey Business School, USA; \\ ${ }^{2}$ Minzu University of China; Department of Finance, School of Economics; China, \\ ${ }^{3}$ Economic Statistics, College of Economics, Jinan University, China.
}

\begin{abstract}
Financial time arrangement, for example, stock cost and trade rates are, regularly, non-straight and non-stationary. Beforehand, numerous scientists have endeavored to conjecture those utilizing factual models and machine learning models. Measurable models expect the time arrangement to be stationary and straight, accordingly bringing about expansive factual mistakes. Examination and expectation of securities exchange time arrangement information has pulled in impressive enthusiasm from the exploration group in the course of the most recent decade. Quick advancement and development of modern calculations for measurable examination of time arrangement information, and accessibility of elite equipment has made it conceivable to prepare and dissect high volume securities exchange time arrangement information successfully, continuously. In monetary field, exceptions speak to unpredictability of securities exchange, which assumes an imperative part in administration, portfolio choice and subordinate evaluating. Along these lines, anticipating anomalies of securities exchange is of the immense significance in principle and application. In this paper, the issue of anticipating anomalies in light of versatile gathering models of Intense Erudition Machines (IEMs) is considered. We discovered that the prescribed novel model is material for exception estimating and beats the strategies in light of auto relapse (AR) Intense Erudition Machines (IEMs) models.
\end{abstract}

Keywords: Stock Intense Erudition Machines (IEMs) models.

\section{Introduction}

Securities exchange is an open business sector for security where sorted out issuance and exchanging of organization stocks occur either through trade or over the counter in physical or electronic structure. It is these days a typical idea that gigantic measures of capital are exchanged through securities exchange all around the globe. Along these lines, precise forecast of securities exchange is exceptionally testing and essential issue for speculators and it has gotten much consideration in money related time arrangement, monetary analysts and specialists. Be that as it may, due to non-straight, non-stationary, profoundly boisterous and confused qualities of securities exchange, the estimating of securities exchange is constantly thought to be extremely troublesome and testing process.

Anomalies can effectsly affect measurable examinations. They can bring about parameter estimation predispositions, invalid derivations and frail unpredictability gauges in money related information. Subsequently when demonstrating monetary information, their discovery and amendment ought to be considered truly. Time-arrangement information is frequently botched up with anomalies because of the impact of strange and non-monotonous occasions. Gauge precision in such circumstances is diminished significantly because of a continue impact of the anomalies on the point conjecture and a predisposition in the appraisal of parameters. Extensive examination has been dedicated to the subject of estimating and different strategies have been recommended which have been separated into two principle bunches: established techniques for the most part exponential smoothing, relapse, Box-Jenkins autoregressive coordinated moving normal, summed up autoregressive restrictively techniques, and cutting edge strategies applying counterfeit consciousness procedures including simulated Semantic systems (SSS) and developmental calculation. Intense Erudition Machines (IEMs) has been prescribed as classes of learning calculation for single concealed 
layer nourish forward neural systems. In Intense Erudition Machines (IEM) calculation, the associations between the information layer and the concealed neurons are haphazardly doled out and stay unaltered amid the learning procedure. Consequently by minimizing the cost capacity through a straight framework the yield associations are tuned. The computational weight of Intense Erudition Machines (IEM) has been altogether lessened as the main expense is tackling a straight framework. The low computational intricacy pulled in a lot of consideration from the examination group, particularly for high dimensional and vast information applications. While extensive examination has been given to identifying and evacuating exceptions, few concentrated on estimating them.

Administration hypothesis has constantly underlined that for any key choice, examining the outside environment is critical. An organization does not exist in segregation. It is a piece of an industry, which itself is inserted in the residential economy. In today's globalized surroundings, the last mentioned, once more, is not invulnerable to world financial developments. The emphasis on the execution of an area is limited to comprehension whether it has solid occasional qualities, or whether it has an overwhelming pattern component, or whether its execution is irregular in nature. It is impeccably feasible for a part to show, overwhelmingly, any of the above three qualities, at various purposes of time. It is vital to screen these examples ceaselessly for both portfolio decisions. Other than giving a system to decay of time arrangement information, for observing the execution of a portfolio, we have likewise composed methodologies for gauging furthermore registered their precision in expectation utilizing reasonably picked preparing and test information sets.

\section{Literature Review}

This study shows an incorporated framework where wavelet changes and repetitive neural system in view of simulated honey bee settlement calculation are consolidated for stock value estimating. The framework involves three phases. To begin with, the wavelet change utilizing the Haar wavelet is connected to break down the stock value time arrangement and in this way take out clamor. Second, the RNN, which has a basic design and uses various major and specialized pointers, is connected to develop the info highlights picked by means of Stepwise Regression-Correlation Selection [1]. In the principal stage, wavelet investigation changes the information space of crude information to a period scale highlight space appropriate for money related anticipating, and after that a Repeated Self-Establishing Map calculation is utilized for parceling and putting away fleeting setting of the component space. In the second stage, numerous piece incomplete minimum square repressors (as neighborhood models) that best fit divided locales are developed for conclusive gauging. Contrasted and neural systems, unadulterated SVMs or conventional GARCH models, the prescribed model performs best. The root-mean-squared estimating mistakes are fundamentally decreased [2].

Monetary time arrangement is nonlinear and non-stationary. Most monetary wonders can't be obviously portrayed in time space. In this manner, customary time space models are not exceptionally compelling in money related estimating. To address the issue, this study consolidates wavelet examination with piece fractional slightest square relapses for stock list anticipating. Wavelet change maps time area inputs to time-recurrence space, where budgetary attributes can be obviously recognized [3].

A successful calculation at foreseeing the stock costs is created. The month to month shutting value information with the Shanghai Composite Index from January 1993 to December 2009 are utilized to represent the utilization of the WDBP neural system based calculation in anticipating the stock record. To demonstrate the benefit of this new approach for stock file conjecture, the WDBP neural system is contrasted and the single Back Propagation neural system utilizing the genuine information set [4-10].

The gauges are assessed with factual criteria, for example, integrity of figure, including tests of conjecture enveloping, directional exactness and the equity of mean squared expectation blunder; the relative estimate execution is surveyed with monetary criteria in a straightforward exchanging system including the effect of exchange expenses on exchanging methodology benefits. Regarding 
measurable criteria, the outcomes demonstrate that distinctive fake neural system particulars gauge superior to the AR model and smooth move non-straight models [11].

Notwithstanding bounteous examination which concentrates on evaluating the level of profit for securities exchange record, there is an absence of studies looking at the consistency of the bearing/indication of stock file development. Given the thought that an expectation with little figure blunder does not as a matter of course interpret into capital increase, we assess the viability of a few multivariate arrangement strategies in respect to a gathering of level estimation approaches. In particular, we lead time arrangement examinations between the two sorts of models on the premise of estimate execution and venture return. The tried arrangement models, which anticipate bearing in view of likelihood, incorporate straight discriminant investigation, logit, probit, and probabilistic neural system. Then again, the level estimation partners, which conjecture the level, are exponential smoothing, multivariate exchange capacity, vector auto relapse with Kalman channel, and multilayered sustain forward neural system. Our near concentrate likewise measures the relative quality of these models as for the exchanging benefit created by their conjectures. To encourage more successful exchanging, we build up an arrangement of limit exchanging rules driven by the probabilities assessed by the order models $[12,13]$.

Another plan in light of tumultuous neural system for stock file forecast is prescribed. The information from a Chinese securities exchange, Shenzhen securities exchange, are connected as a contextual analysis. The riotous neural system is utilized to take in the non-straight stochastic and disordered examples in the stock framework and estimate another list with previous records. The legitimacy of the plan is investigated hypothetically, and the recreation results demonstrate that it has a decent execution $[14,15]$.

\section{Methodology}

Outline of the Recommended Forecasting Technique

The prescribed expectation framework comprises of three stages: (1) The authentic stock value time arrangement $\mathrm{o}(\mathrm{t})$ are prepared with a discrete wavelet change; (2) Both guess $\mathrm{A}(\mathrm{t})$ and point of interest $\mathrm{D}(\mathrm{t})$ coefficients are removed to shape the primary element vector and (3) The framed element vector nourishes the contribution of a back-spread neural system.

\section{Recommended Approach}

\subsection{Wavelet Transforms.}

This area contains a few realities about wavelets, utilized all through this paper. A careful audit of the wavelet change is talked about. Wavelet examination dissects limited varieties of sign inside a period arrangement. Both the prevailing methods of variability and their varieties in time can be caught by disintegrating a period arrangement into time-scale space. Discrete Wavelet Transform (DWT) can deteriorate the sign in both time and recurrence area at the same time. Then again, Fourier Transform breaks down the sign just in recurrence space; data identified with event of recurrence is not caught and it disposes of the time determination. The wavelet investigation is a scientific device that offers decay of sign $\mathrm{r}(\mathrm{u})$ into numerous recurrence groups at numerous scales. Specifically, the sign $r(u)$ is deteriorated into smooth coefficients $s \alpha$ and point of interest coefficients $d \alpha$, which are given by the following formulas.

$$
\begin{aligned}
& s \alpha_{a, b}=\int r(u) \emptyset_{a, b}(u) d t \\
& d \alpha_{a, b}=\int r(u) \Psi_{a, b}(u) d t
\end{aligned}
$$

Where $\Phi$ is the father and $\Psi$ is the mother wavelets, and $\mathrm{j}$ and $\mathrm{k}$ are, individually, the scaling and interpretation parameters. The father wavelet keeps the recurrence area property that is low-recurrence of the sign, while the mother wavelet keeps the time space property that is 
high-recurrence. The decay of sign utilizing DWT is appeared as a part of Figure 1. DWT works on the guideline of convolution.

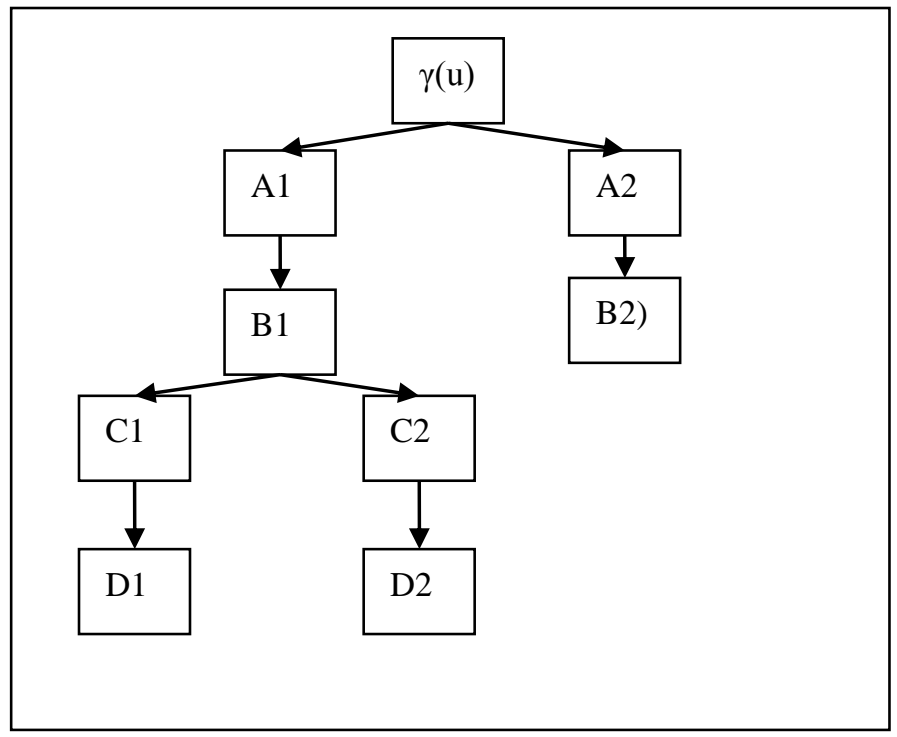

Figure 1 DWT for Decomposition of Signal

Different sorts of wavelets are accessible, for occurrence. Morlet and Daubechies wavelets have applications in picture handling and are liable to associating issue. Mexican Hat wavelets are costly to figure. Haar wavelets are favorable for time arrangement examination since they are fit for catching variances between nearby perceptions. One of the confinements of DWT is the prerequisite of length of the dataset to be dyadic. Besides, the yield produced by DWT is very subject to inception of the sign being examined. A little move in birthplace influences the yields created and this issue is called Circular movement. Because of round movement, it is hard to adjust the changed signs to time.

\subsection{Simulated Semantic Systems (SSS).}

Enlivened by organic neural systems (cerebrum), Simulated Semantic Systems (SSS) are group of models in machine discovering that have been created, and are utilized for capacity estimation, arrangement and relapse. SSS model comprise of thickly interconnected computational units called neurons. The neurons are sorted out in three layers: info layer, shrouded layer and yield layer. The association between neurons of back to back layers is weight. The principal layer in the system, info layer relates to information variables. The second layer in the system is shrouded layer, which is associated with both info and yield layer. The neurons in the concealed layer perform two operations: (i) they get data sent from the neurons in the info layer; and (ii) increase the data by a weight variable. Weighted data of every single approaching association is included every neuron of the shrouded layer lastly an inclination term is incorporated. The summed info and inclination are handled utilizing a non-direct capacity (sigmoid or hyperbolic digression capacity) to get a yield worth which is then sent to the neurons in the yield layer, the third layer of the system.

\subsection{Intense Erudition Machines (IEM) Algorithm.}

The reason for this paper is to examine the mythology behind the Intense Erudition Machines (IEM). Intense Erudition Machines (IEM) is an enhanced learning calculation for the single food forward neural system structure. It eminently varies from the conventional neural system philosophy, since it is not key to tune every one of the parameters of the food forward systems (info weights and shrouded layer inclinations). For more data on productivity of SLFNs with arbitrarily picked information weights, concealed layer inclinations and a nonzero actuation capacity to rough any persistent capacities on any info set. The prescribed Intense Erudition Machines (IEM) has demonstrated its effectiveness in preparing nourish forward neural systems and defeating the impediments confronted by other ordinary calculations.

The characters of Intense Erudition Machines (IEM) lie in two viewpoints, that is, irregular neurons and the without tuning technique. The learning period of Intense Erudition Machines (IEM) for the most part incorporates two stages, specifically, developing the concealed layer yield network 
with irregular shrouded neurons and finding the yield associations. On account of utilizing irregular concealed neuron parameters which stay unaltered amid the learning stage, Intense Erudition Machines (IEM) appreciates a low computational multifaceted nature. The computational weight has been extraordinarily lessened as the main expense is tackling a direct framework. In the meantime, various applications have demonstrated that Intense Erudition Machines (IEM) can give a tantamount or preferable speculation execution over the mainstream bolster vector machine (SVM) and the BP technique by and large.

\section{Model and Feature Selection}

Suggested Hybrid Models

The recommended fractious kind representations (recommended fractious kind representations -SSS and recommended fractious kind representations - Support Vector Regression) for expectation of stock cost incorporate the benefits of a disintegration model and machine learning models.

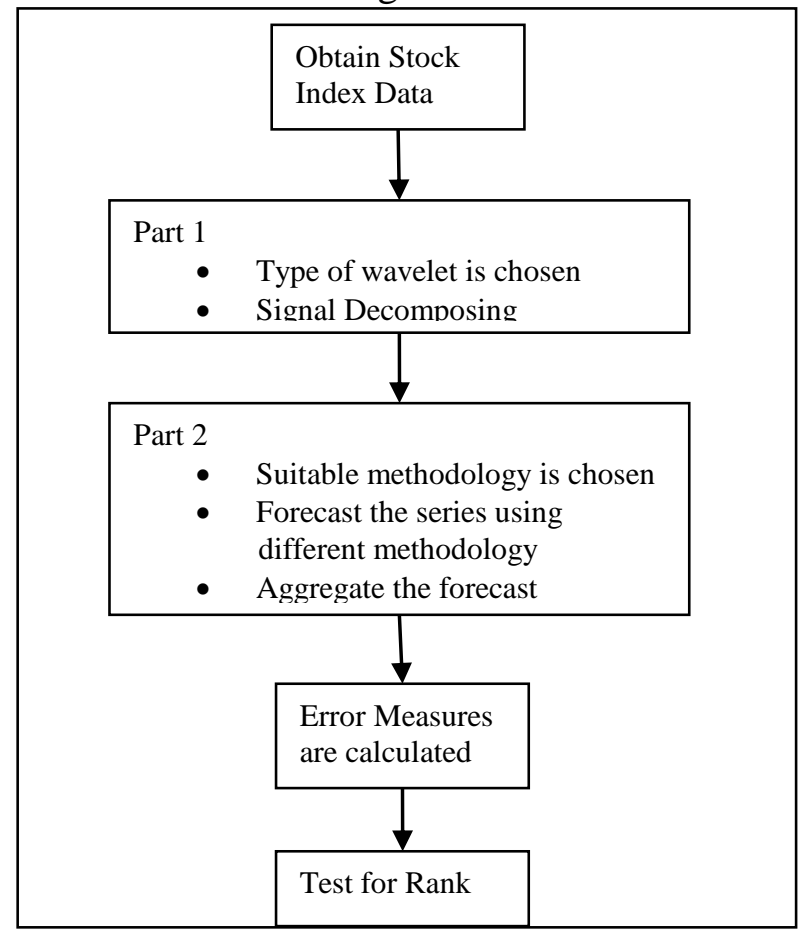

Figure 2. Flow Chart of the Recommended Hybrid Models

The strides are counted beneath:

(1) The first arrangement is deteriorated into an arrangement of different sub-arrangement utilizing DWT.

(2) Every sub-arrangement is determined independently utilizing SSS and Support Vector Regression as a part of instance of the mixture recommended fractious kind representations -SSS and recommended fractious kind representations - Support Vector Regression models, individually.

(3) Determined sub-arrangement is recombined to get the last conjecture esteem.

(4) Execution/blunder measures are figured by looking at the last estimate esteem (got in the past stride) with the first arrangement.

(5) To comprehend the viability of the prescribed models, Wilcoxon-Signed Rank Test, a non-parametric measurable model is utilized to analyze the prescient ability of recommended fractious kind representations -SSS and recommended fractious kind representations - Support Vector Regression with SSS and Support Vector Regression models (without decay).

The period considered for the study covers the 2008 Financial Crisis. The world economy was influenced. The explanations behind decay of securities exchange are cited as:

(i) Troubles were experienced while bringing reserves up in the household market,

(ii) Foreign Institutional Investor assets were pulled back from the securities exchanges, 
(iii) Credit smash in banks. The execution of National Stock Exchange of USA record is appeared in Figure 3.

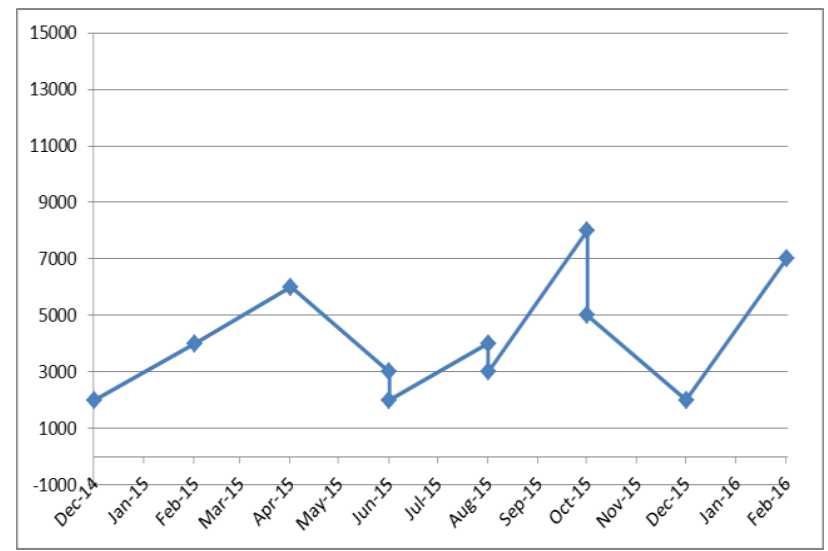

Figure 3 Performance of National Stock Exchange Index during 2015 Financial Crisis

\section{Conclusion and Future Work}

The paper suggested and exhibited a half and half determining approach coordinating the benefits of a deterioration model and machine learning models. The exhibited models demonstrated a steady prevalent execution in anticipating the week by week National Stock Exchange Fifty list, when contrasted with both SSS and Support Vector Regression, which show their capacity to conjecture non-stationary and non-straight time arrangement. From the analyses, it can be watched that decay of the arrangement utilizing recommended fractious kind representations helped as a part of enhancing the execution of the machine learning models altogether. The execution measures acquired emphasize the certainty the estimates got by amassing the anticipated parts of a period arrangement are more exact than the figures got by demonstrating the first arrangement itself.

The suggested model is worthwhile for anticipating the stock list since it is a disintegration based model. It deconstructs the time-arrangement into different recurrence parts. Singular parts are less demanding to anticipate than unique non-stationary and non-straight information arrangement. The second part of the model uses Support Vector Regression which has powerful execution than SSS model. Since the prescribed model is information versatile model, the pattern in the time arrangement is caught and this aides in expanding the precision of the forecast.

\section{References}

[1] Hsieh T-J, Hsiao H-F, Yeh W-C. Forecasting stock markets using wavelet transforms and recurrent neural networks: an integrated system based on artificial bee colony algorithm. Applied Soft Computing. 2011; 11(2):2510-25.

[2] Huang SC, Wu TK. Integrating recurrent SOM with wavelet-based kernel partial least square regressions for financial forecasting. Expert Systems with Applications. 2010; 37(8):5698-705.

[3] Huang SC. Forecasting stock indices with wavelet domain kernel partial least square regressions. Applied Soft Computing. 2011; 11(8):5433-43.

[4] Wang J Z, Wang JJ, Zhang ZG. Guo SP. Forecasting stock indices with back propagation neural network. Expert Systems with Applications. 2011; 38(11):14346-55.

[5] Chen, Y., Dong, X. \& Zhao, Y., "Stock Index Modeling Using EDA Based Local Linear Wavelet Neural Network", Proceedings of International Conference on Neural Networks and Brain, pp. 1646-1650, 2005. 
[6] Chen, A.-S., Leung, M. T. \& Daouk, H., "Application of Neural Networks to an Emerging Financial Market: Forecasting and Trading the Taiwan Stock Index", Operations Research in Emerging Economics Vol 30, No 6, pp. 901- 923, 2003.

[7] Hanias, M., Curtis, P. \& Thalassinos, J., "Prediction with Neural Networks: The Athens Stock Exchange Price Indicator", European Journal of Economics, Finance and Administrative Sciences, Vol 9, pp. 21-27, 2007.

[8] de Faria, E., Albuquerque, M. P., Gonzalez, J., Cavalcante, J. \& Albuquerque, M. P., "Predicting the Brazilian Stock Market through Neural Networks and Adaptive Exponential Smoothing Methods", Expert Systems with Applications, 2009.

[9] Wu, Q., Chen, Y. \& Liu, Z., "Ensemble Model of Intelligent Paradigms for Stock Market Forecasting" Proceedings of the IEEE 1st International Workshop on Knowledge Discovery and Data Mining', Washington, DC, USA, pp. 205-208, 2008.

[10] Wang, W. \& Nie, S., "The Performance of Several Combining Forecasts for Stock Index", International Workshop on Future Information Technology and Management Engineering, pp. , 450-455, 2008.

[11] Perez-Rodriguez, J. V., Torra, S. \& Andrada-Felix, J., 'Star and ANN Models: Forecasting Performance on the Spanish IBEX-35 Stock Index', Journal of Empirical Finance, Vol 12, No 3, pp. 490-509, 2005.

[12] Leung, M. T., Daouk, H. \& Chen, A.-S., "Forecasting Stock Indices: A Comparison of Classification and Level Estimation Models", International Journal of Forecasting Vol 16, No 2, pp. 173-190, 2000.

[13] Kim, K.-J., “Artificial Neural Networks with Feature Transformation Based on Domain Knowledge for the Prediction of Stock Index Futures", Intelligent Systems in Accounting, Finance \& Management, Vol 12, No 3, pp. 167-176, 2004.

[14] Ning, B., Wu, J., Peng, H. \& Zhao, J., 'Using Chaotic Neural Network to Forecast Stock Index', Advances in Neural Networks, Vol 5551, pp.870-876, 2009.

[15]Pan, H., Tilakaratne, C. \& Yearwood, J., 'Predicting the Australian Stock Market Index Using Neural Networks Exploiting Dynamical Swings and Intermarket Influences', Journal of Research and Practice in Information Technology, Vol 37, No 1, pp. 43-55, 200 